\title{
Comparative Evaluation of the Shear Bond Strength and Debonding Properties of a Conventional Composite and Flowable Composites used for Orthodontic Bracket Bonding
}

\author{
S Pradeep, Ravi Shanthraj, H Jyothi Kiran, BM Shivalinga
}

\begin{abstract}
Aim: To evaluate and compare shear bond strength (SBS) and debonding characters of the Transbond XT (BisGMA-based composite), Esthet-X flow (flowable composite), Filtek Z-350 (flowable composite).
\end{abstract}

\begin{abstract}
Materials and methods: A total of 90 human premolars were divided into group I, Transbond XT $(n=30)$; group II, Esthet-X flow $(n=30)$ and group III, Filtek Z-350 $(n=30)$, the preadjusted edgewise stainless steel premolar brackets were bonded to evaluate the shear bond and debonding properties.
\end{abstract}

Results: The results of the statistical analysis comparing the three groups indicated no statistically significant differences between the groups. In general, the SBS with groups I and III exhibited similar bond strength with $11.58 \pm 1.3 \mathrm{MPa}$ and $11.07 \pm$ 1.0 MPa respectively. Groups III exhibited least bond strength of $10.7 \pm 2 \mathrm{MPa}$. In modified adhesive remnant index (ARI) the majority of bond failures occurred at enamel-adhesive interface or cohesive type failure in all the three groups. Groups II and III showed increased frequency of score 2, 4 and 5 compared to group I which showed increased frequency of score 0 and 1.

Conclusion: When considering the SBS and ARI scores obtained, flowable composites can be effectively applied to orthodontic bracket bonding.

Keywords: Shear bond strength, Esthet-X flow, Filtek Z-350, Flowable composites, Etching, Bonding, Brackets, Adhesives.

How to cite this article: Pradeep S, Shanthraj R, Kiran HJ, Shivalinga BM. Comparative Evaluation of the Shear Bond Strength and Debonding Properties of a Conventional Composite and Flowable Composites used for Orthodontic Bracket Bonding. World J Dent 2013;4(1):6-16.

Source of support: Nil

Conflict of interest: None declared

\section{INTRODUCTION}

The introduction of acid etch technique by Buonocore ${ }^{1}$ in 1955 heralded a new era in adhesive dentistry, which has since initiated varied applications in the field of dentistry including bonding of orthodontic attachments. ${ }^{2,3}$

Newman in 1965 introduced the novel concept of bonding orthodontic attachments to tooth surfaces by means of epoxy adhesive. This procedure improved the overall treatment results by eliminating band occupying interdental spaces, decreased gingival irritation, and easier removal of plaque and decreased risk of calcification. ${ }^{2}$ Since then, various dental adhesives and methods of bonding orthodontic attachments have been reported to enhance the bond strength of the orthodontic attachments by pretreatment of enamel surfaces. ${ }^{3-5}$

Previous generation bonding systems used conventional adhesives comprising of three different agents, an enamel conditioner, a primer solution, and an adhesive resin during the bonding of orthodontic brackets to enamel. ${ }^{6}$

Revolutionary advances in adhesive chemistry are changing the process of orthodontic bonding. ${ }^{10}$ The constant quest for better bonding systems to reduce the technique sensitivity of the adhesion procedures, to improve the bond strength, to reduce the loss of enamel and to reduce the number of clinical application steps as well as chairside time has resulted in innovation of many bonding agents. ${ }^{6-10}$ Fewer steps in the bonding process mean fewer human errors.

Though composite resin has wide clinical acceptance both for bonding of brackets or restorative purpose, several drawbacks have been reported which include loss of enamel during acid etching and debonding, enamel decalcification around brackets and lowered bond strength in the presence of water or moisture.

Incomplete polymerization. ${ }^{8,11,12}$ even when mixed and cured according to manufacturer's instructions is another problem of composite resin resulting in residual monomer that is readily leached from the cured resin. Adverse reactions caused by leaching of residual monomer have also been reported based on the in vitro and in vivo studies on biocompatibility, potential mutagenicity, ${ }^{13,14}$ and estrogenicity of various orthodontic adhesives. Recently, it has been reported that bisphenol $\mathrm{A}$, a component in the structure of bisphenol-a-glycidyl dimethacrylate (BisGMA) exhibits estrogenicity ${ }^{15}$ and BisGMA itself has been found to be cytotoxic in a number of cell culture studies. ${ }^{16,17}$ The presence of residual methacrylate monomer has been blamed for elution of toxic reactants.

As an alternative to traditional BisGMA-based composite resins, newer bonding adhesives like glass ionomer cement (GIC), resin-modified GIC, compomers and flowable composites have been developed and tried with varying degrees of success. 
Thus, the flow property is an important feature for clinical consideration that influences both the penetration of the adhesive into retentive mechanism at the bracket base and the ability of an adhesive to resist bracket drift during direct bonding. Hence, among the various innovations introduced in composite resins, a plethora of low viscous composite resins and flowable composites were marketed for restorative purpose and bonding orthodontic attachments which needs special mention. Flowable composites are low viscosity composite resins, created by retaining the same particle size of traditional hybrid composites, but reducing filler content and increasing resin content to reduce viscosity of resin.

Flowable composites were marketed for bonding of brackets during early 21st century. Flowable composite merits special attention because of their clinical handling characters of nonstickiness, fluid injectability ${ }^{18}$ and shear bond strength (SBS) comparable to that of traditional composite adhesives. ${ }^{19}$

Considering the merits and demerits of BisGMA-based composite resin, and flowable composite the present in vitro study was planned to evaluate Esthet-X flow, Filtek Z-350 flowable composites as an alternative orthodontic bonding agent by determining its SBS and debonding characters and to compare it with that of conventionally used BisGMA-based composite resin Transbond XT composite.

\section{MATERIALS AND METHODS}

The present in vitro study was planned to evaluate and compare the SBS of a conventional composite and flowable composites used for orthodontic bracket bonding.

Stainless steel metal brackets were bonded on extracted human premolar teeth using the above three mentioned adhesives. SBS and debonding characters of the three materials under study were determined and compared. The following materials and equipment were used for the above study:

I. Materials

- Adhesive system for evaluation:

- Transbond XT (3M UNITEK, Monrovia, California) (Fig. 1A)

- Esthet-X flow (Dentsply) (Fig. 1B)

- Filtek Z-350 (3M UNITEK, Monrovia, California) (Fig. 1C)

- Preadjusted edgewise upper premolar stainless steel brackets (Gemini, 3M Unitek, Monrovia, California) (Fig. 1D)

- Upper and lower premolar teeth extracted for therapeutic purpose (Fig. 1E)

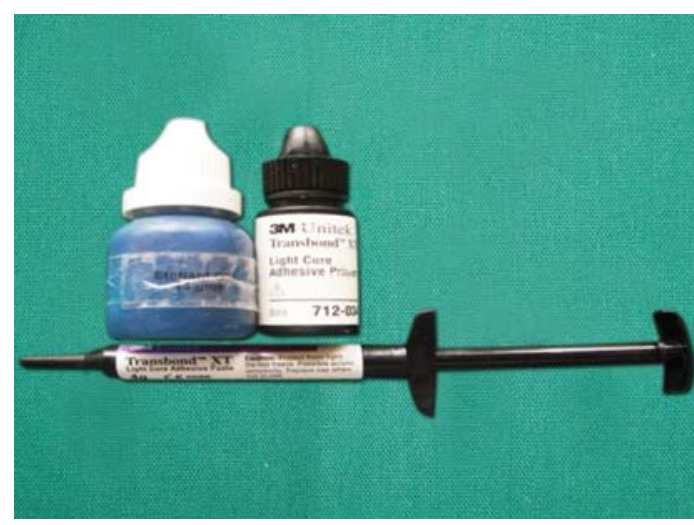

Fig. 1A: Transbond XT

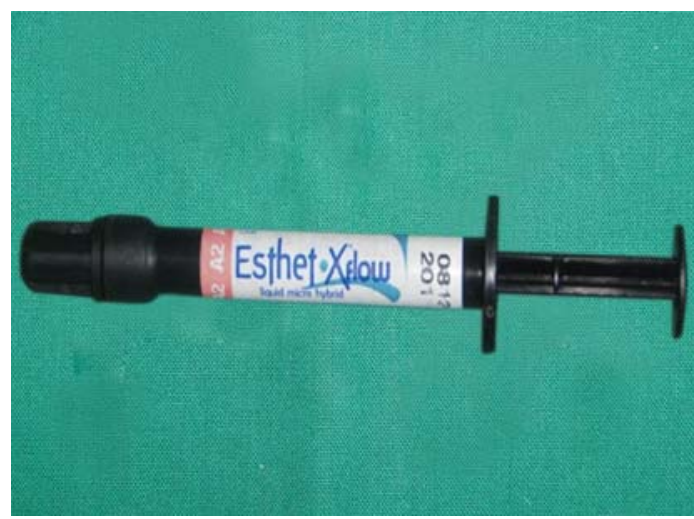

Fig. 1B: Esthet-X flow

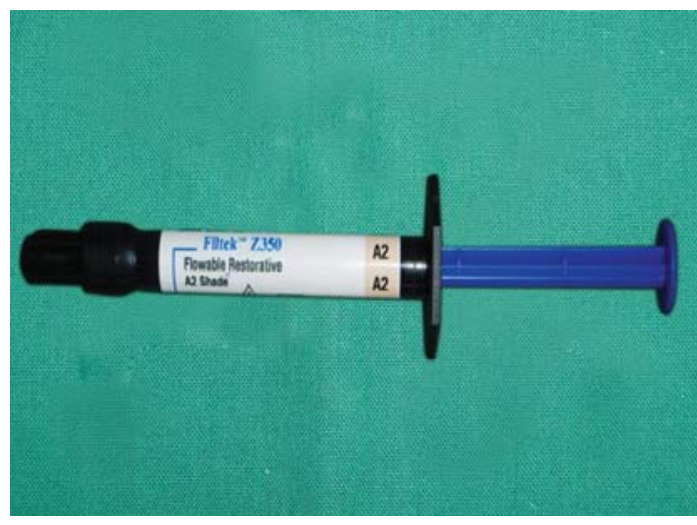

Fig. 1C: Filtek Z-350

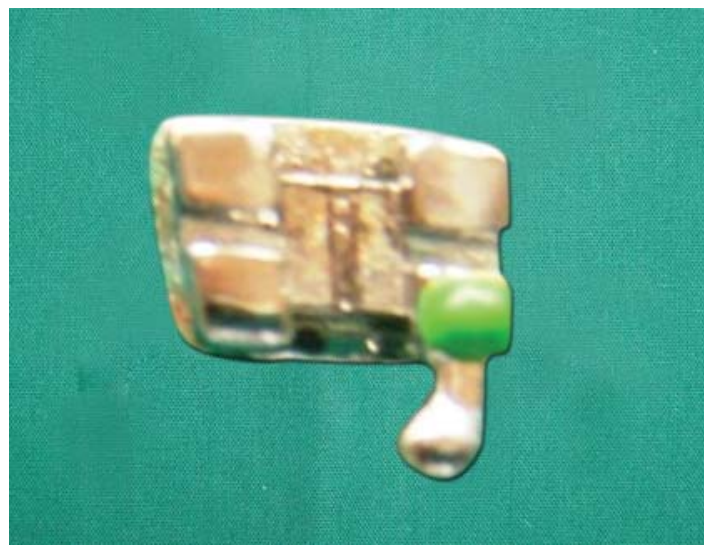

Fig. 1D: Premolar bracket 


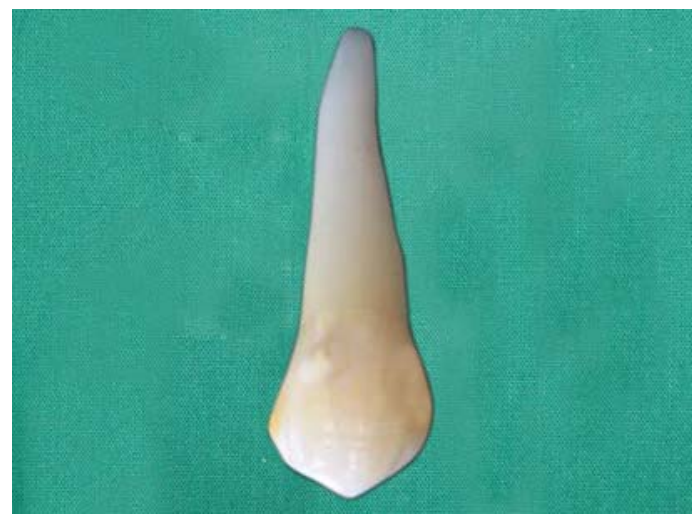

Fig. 1E: Extracted upper premolar tooth

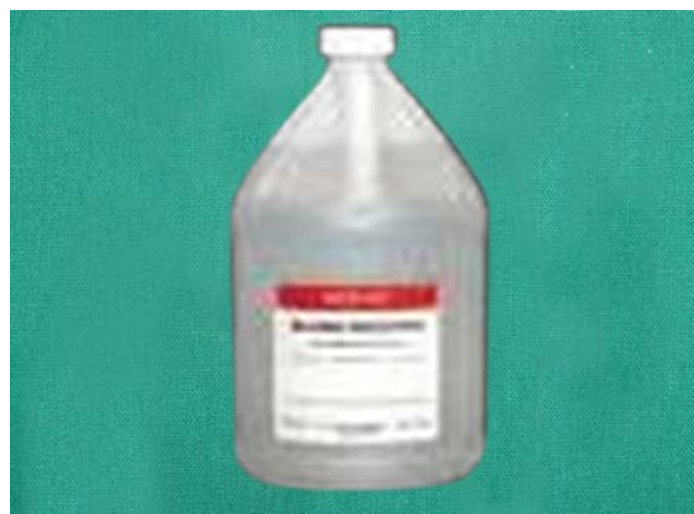

Fig. 1F: Thymol solution

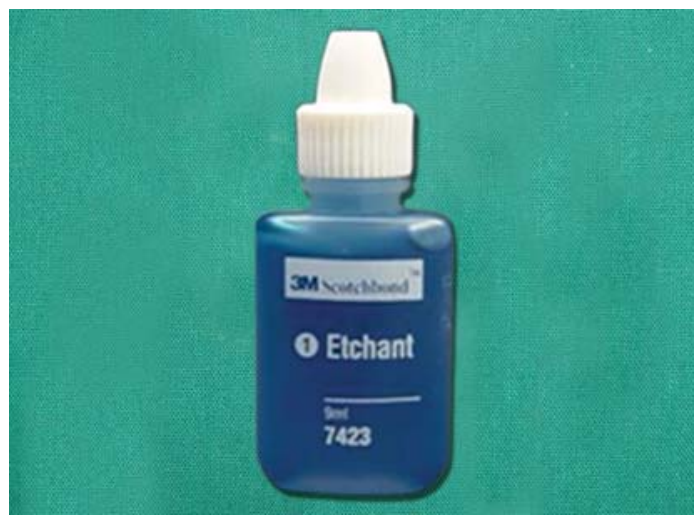

Fig. 2A: Etchant-37\% phosphoric acid

- Thymol solution for storing the teeth before bonding (Fig. 1F).

II. Bonding accessories

- Etchant: 37\% phosphoric acid (Scotch bond 3M ESPE, MN) (Fig. 2A)

- Applicator brush (Fig. 2B)

- 3-way syringe (Fig. 2C)

- Bracket holder (Fig. 2D)

- Bracket positioner (Fig. 2E)

- Explorer (Fig. 2F)

- Light emitting diode curing unit-Starlight P (Monitex Bluelex) (Fig. 2G)

- $\quad$ Polishing rubber cup and pumice powder (Fig. 2H).

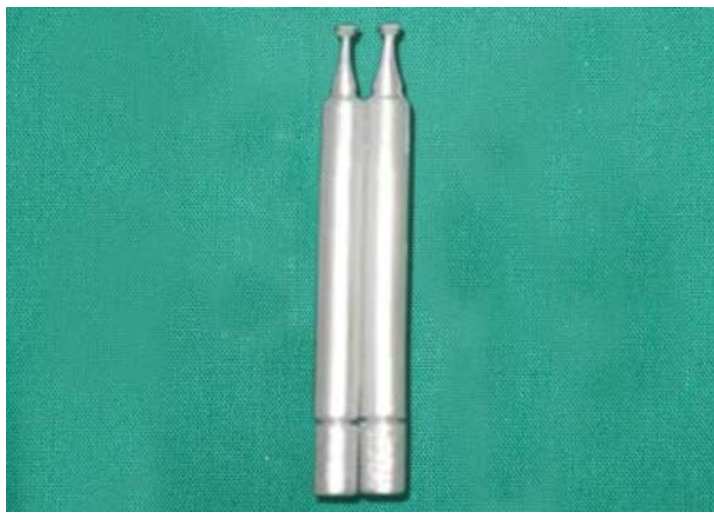

Fig. 2B: Applicator brush

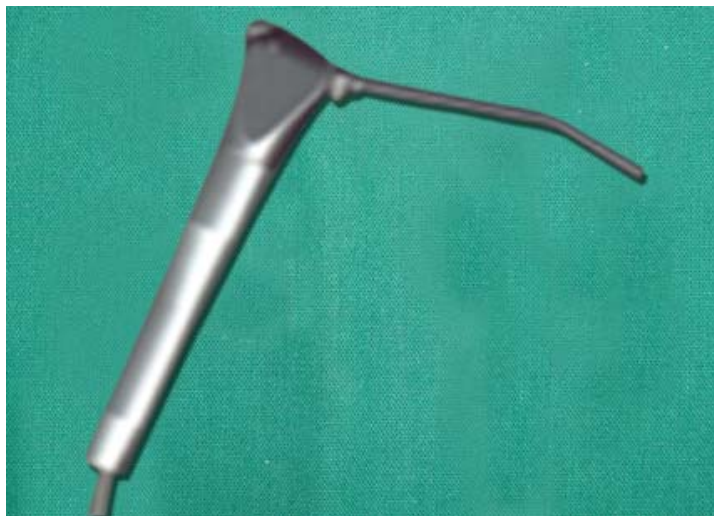

Fig. 2C: 3-way syringe

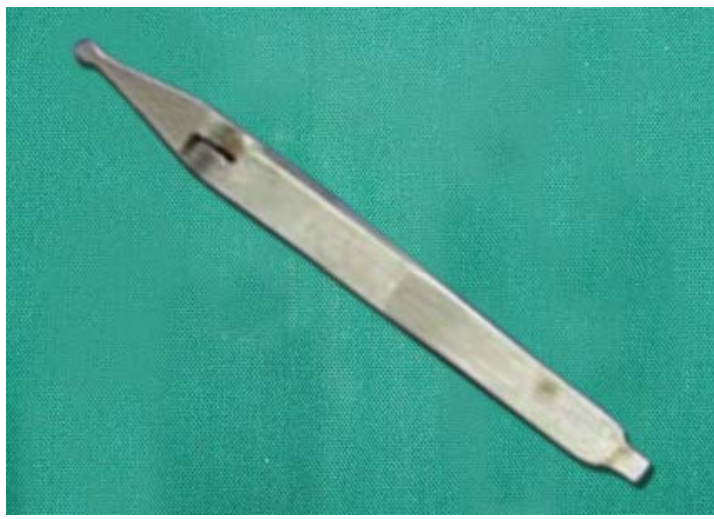

Fig. 2D: Bracket holder

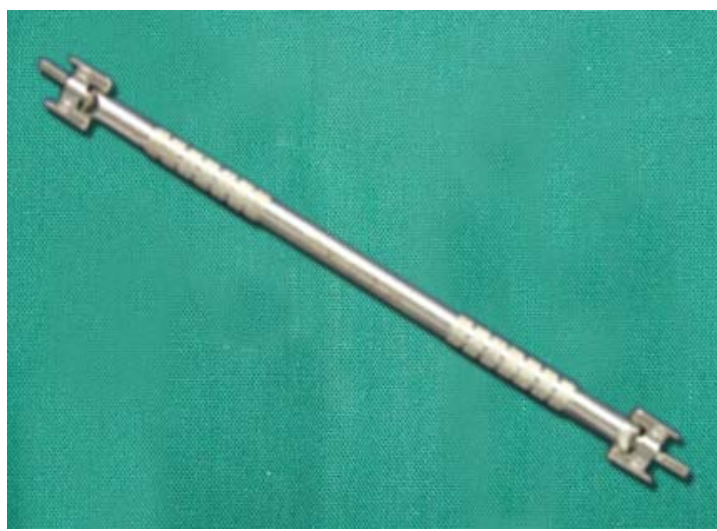

Fig. 2E: Bracket positioner 


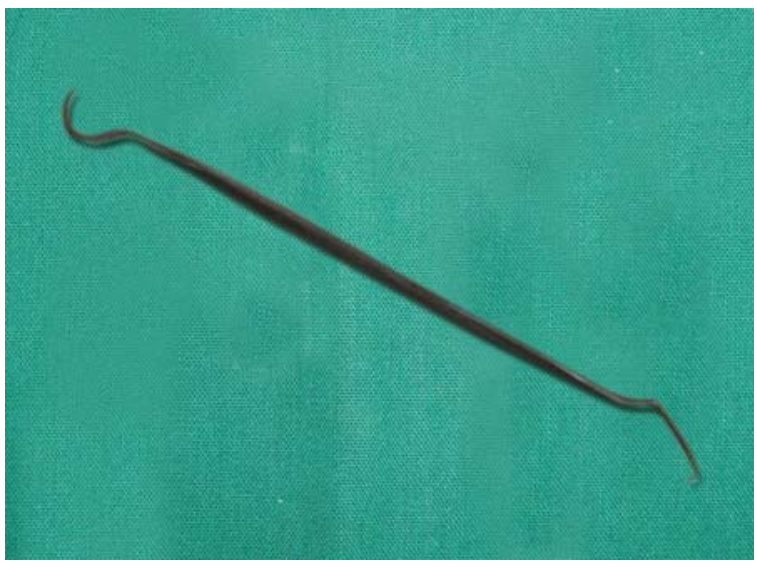

Fig. 2F: Explorer

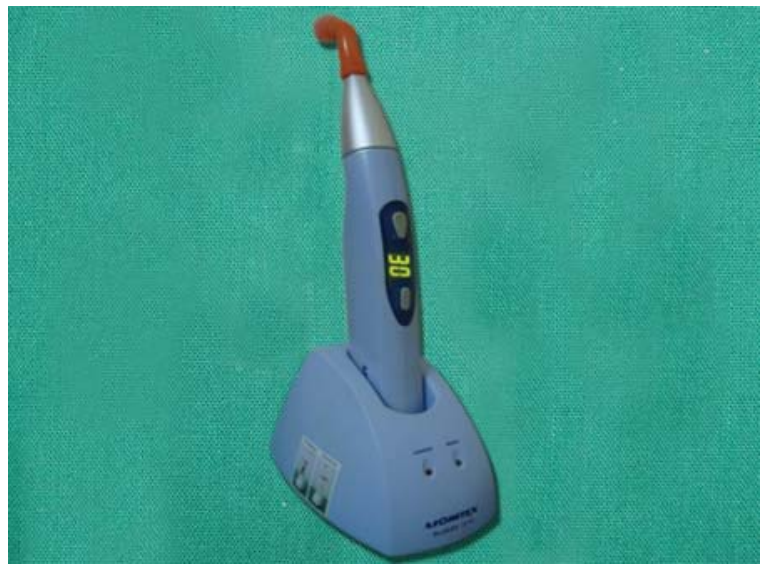

Fig. 2G: Polishing rubber cup and pumice powder

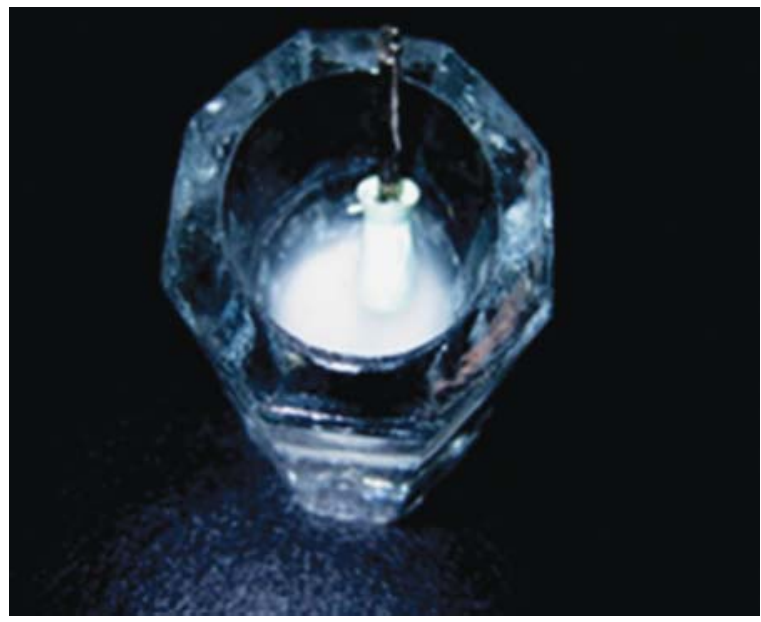

Fig. 2H: Polishing rubber cup and pumice powder

III. Color coded of the acrylic blocks

- Group 1 - Transbond XT: Green (Fig. 3A)

- Group II - Esthet-X flow: Black (Fig. 3B)

- Group III - Filtek Z-350: Blue (Fig. 3C)

IV. Equipments: Used to evaluate SBS

- Universal testing machine JJ LLOYD 20 KN (Fig. 4A)

- Custom-made wooden jig (Fig. 4B)

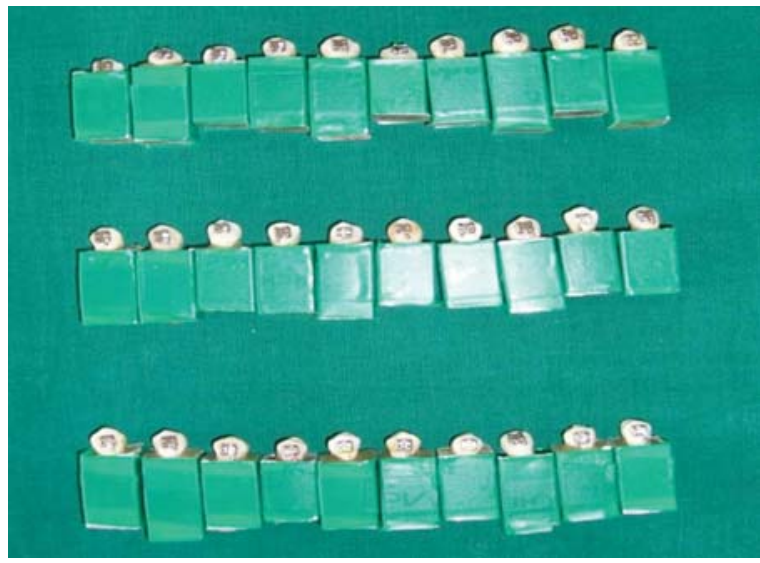

Fig. 3A: Group I-Transbond XT (green)

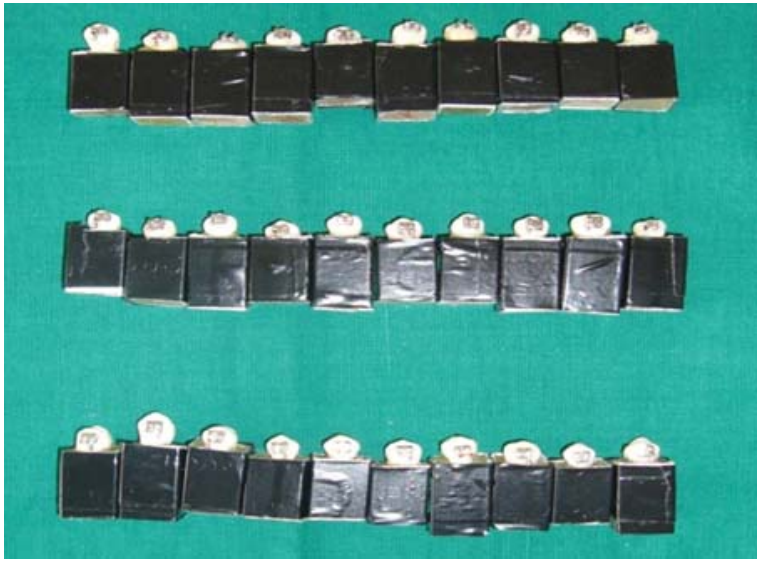

Fig. 3B: Group II-Esthet-X flow (black)

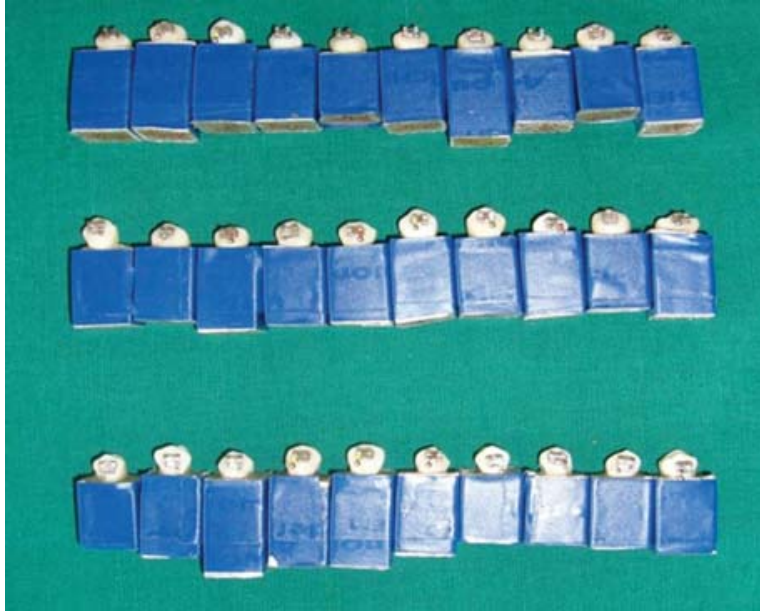

Fig. 3C: Group III-Filtek Z-350 (blue)

- Aluminum block with tooth embedded attached to lower jaw of testing machine with custom-made zig and chisel mounted to upper jaw (Fig. 4C)

- Digital Vernier calipers (Fig. 4D)

V. Equipments: Used to evaluate debonding characters

- Optical microscope (Fig. 5A)

- Scanned bracket base (Figs 5B to G). 


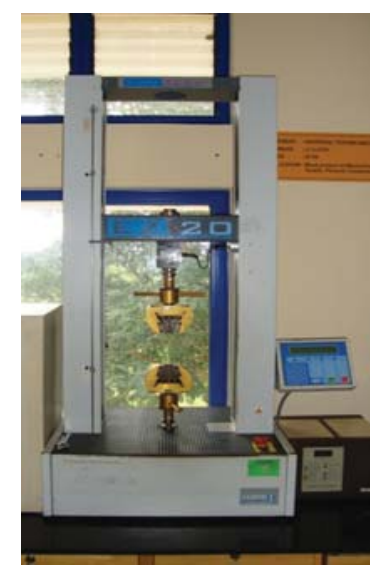

Fig. 4A: JJ Lloyd testing machine

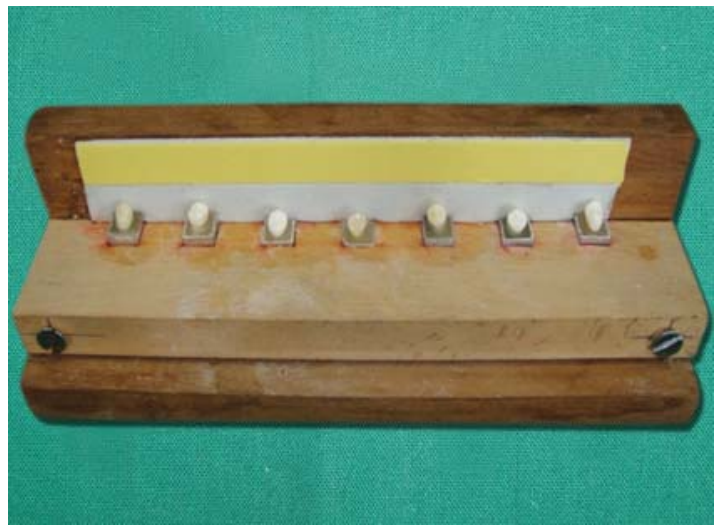

Fig. 4B: Custom-made wooden zig

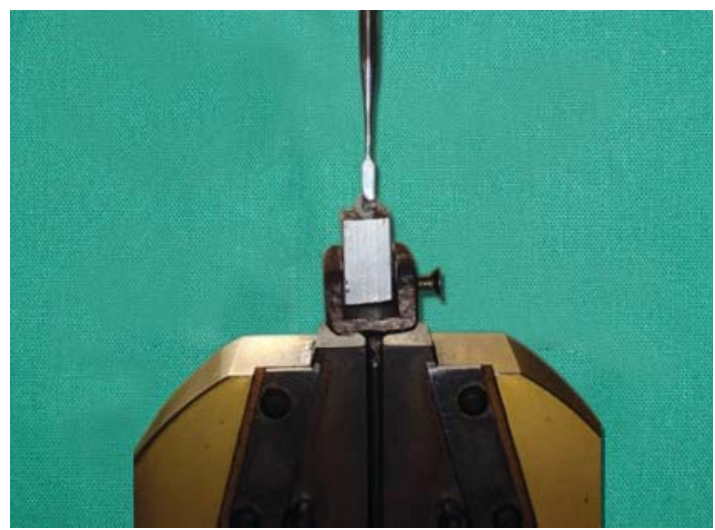

Fig. 4C: Aluminum block with tooth embedded attached to lower jaw of testing machine with custom-made zig and chisel mounted to upper jaw of testing machine

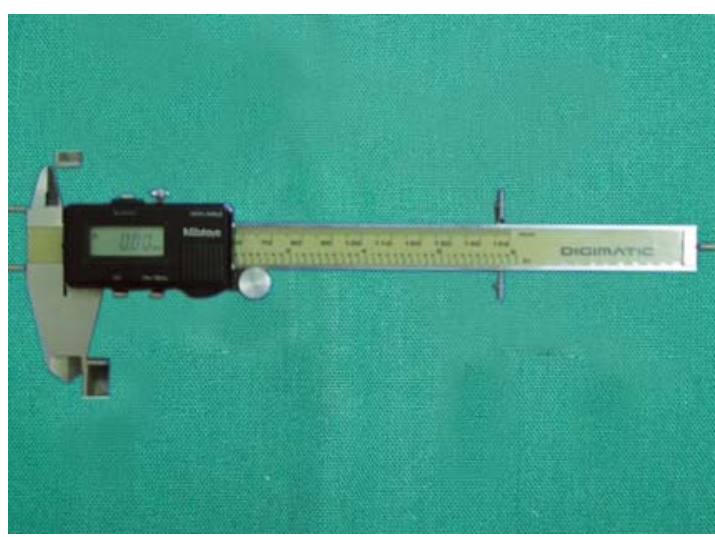

Fig. 4D: Digital vernier calipers used for measuring the base of the bracket

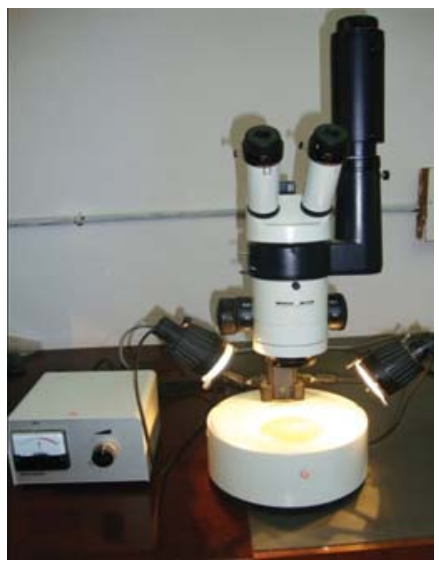

Fig. 5A: Stereomicroscope

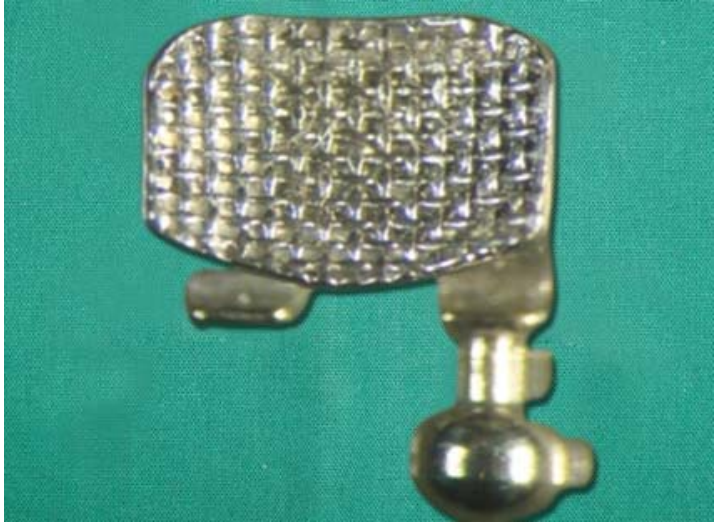

Fig. 5B: $A R I$ score $=0$

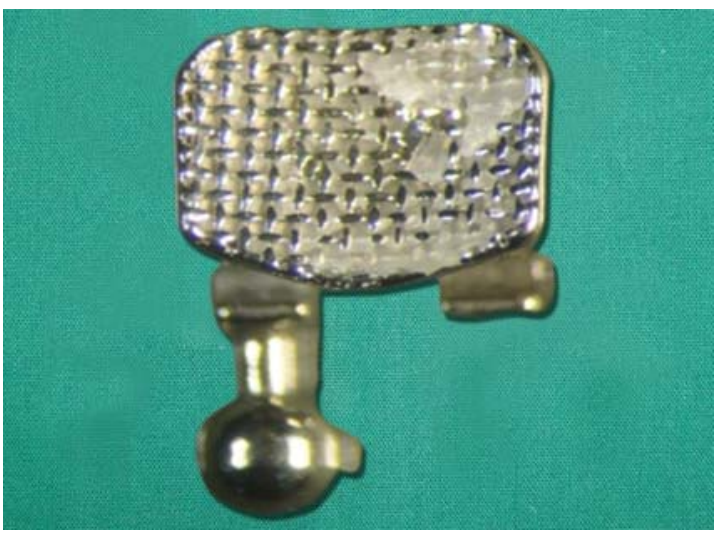

Fig. 5C: $A R I$ score $=1$

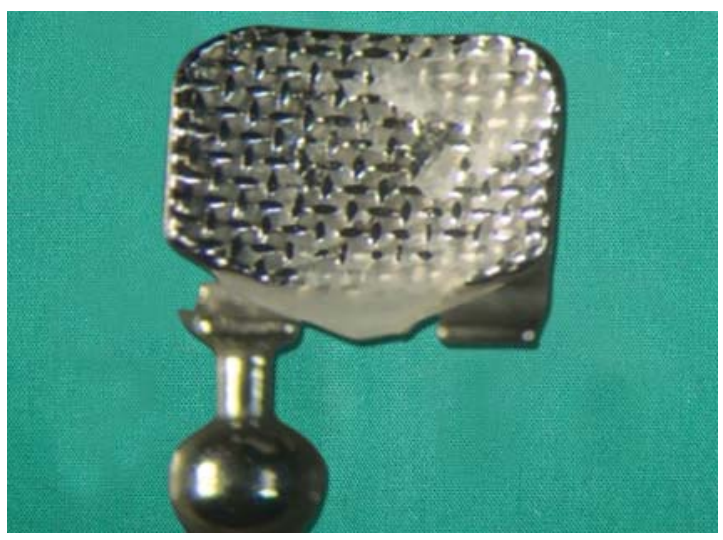

Fig. 5D: ARI score $=2$ 


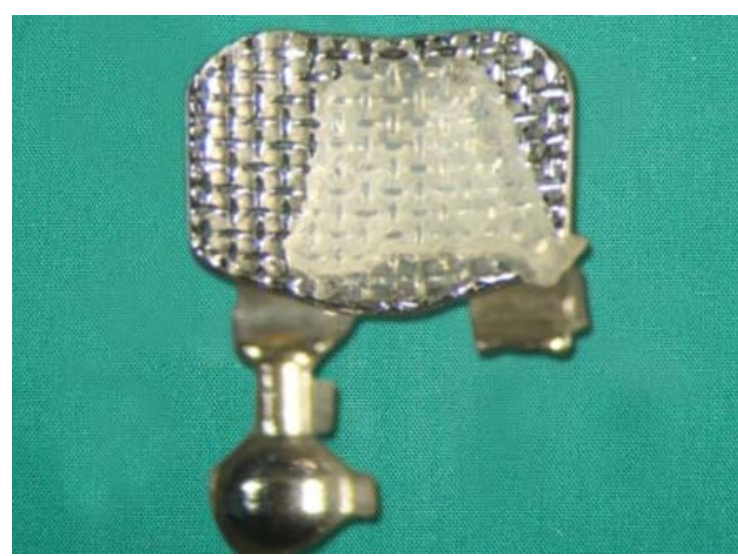

Fig. 5E: ARI score $=3$

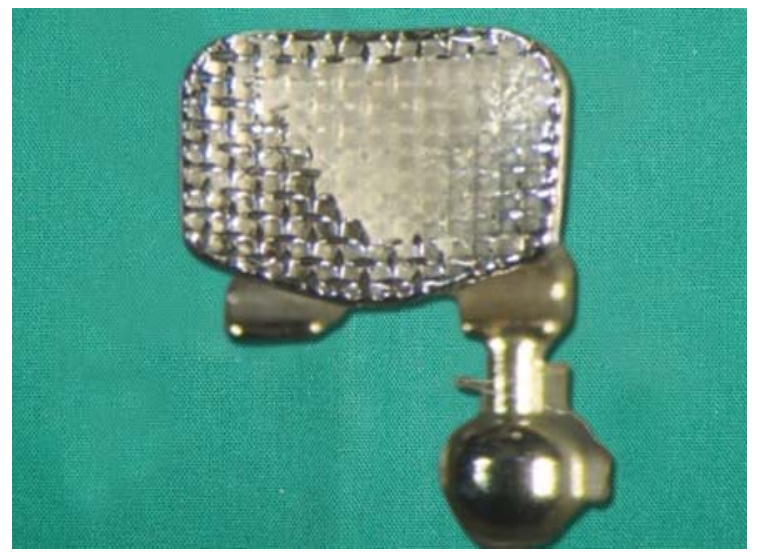

Fig. 5F: ARI score $=4$

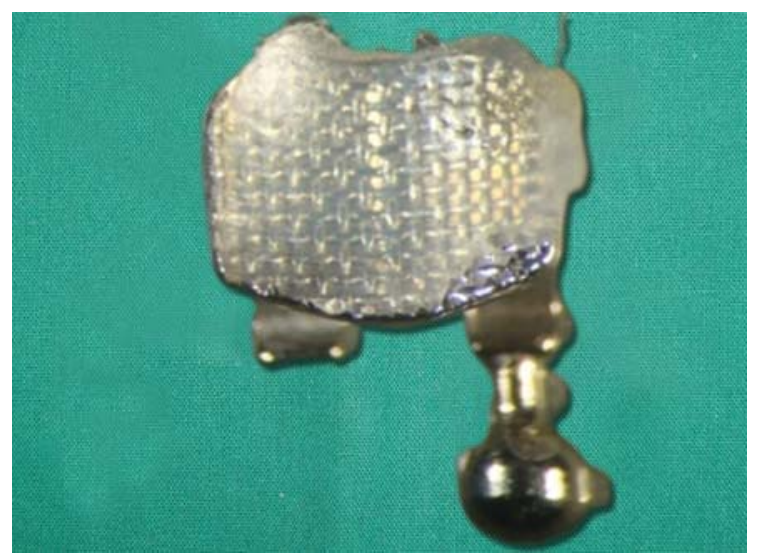

Fig. 5G: ARI score $=5$

\section{METHODOLOGY}

Sample for the study: Ninety preadjusted edgewise upper premolar stainless steel brackets (Gemini 3M Unitek) and 90 freshly extracted premolar teeth were used in the study.

Ethical committee clearance was obtained from the ethical committee, JSS University, for the use of natural teeth for the study.

Inclusion criteria for the teeth to be evaluated in the study are:

- Anatomically and morphologically well-defined teeth

- Noncaries maxillary and mandibular premolar teeth with intact buccal enamel, extracted for orthodontic purpose
Exclusion criteria for the sample are as follows:

- Teeth with heavy restorations

- Variations in crown morphology with enamel structural defect

- Teeth having surface cracks from extraction forceps

- Teeth treated with chemical agents

- Fluorosed teeth.

Sample preparation and distribution: The freshly extracted teeth were cleaned to remove blood or any tissue debris and stored in $0.1 \% \mathrm{wt} / \mathrm{vol}$ thymol solution to prevent bacterial contamination and dehydration.

The teeth were divided into three groups of 30 each to be bonded with stainless steel metal brackets using three different adhesives under study.

The teeth were then mounted on self-cured, colored blocks of dimensions $25 \times 10 \times 10 \mathrm{~mm}$ such that the roots were completely embedded into the acrylic block up to cementoenamel junction and the buccal surface of crown perpendicular to base of the block. The acrylic blocks were color coded to differentiate between different groups.

The teeth to be bonded with stainless steel metal brackets using three adhesives under study were grouped as given below (Figs 3A to C).

\begin{tabular}{llc}
\hline $\begin{array}{l}\text { Color coding of } \\
\text { aluminum blocks }\end{array}$ & $\begin{array}{l}\text { Adhesive used } \\
\text { for bonding }\end{array}$ & $\begin{array}{l}\text { No. of metal } \\
\text { brackets (sample } \\
\text { size) }\end{array}$ \\
\hline $\begin{array}{l}\text { Green: Group I } \\
\text { (Fig. 3A) }\end{array}$ & Transbond XT & 30 \\
\hline $\begin{array}{l}\text { Black: Group II } \\
\text { (Fig. 3B) }\end{array}$ & Esthet-X flow & 30 \\
\hline $\begin{array}{l}\text { Blue: Group III } \\
\text { (Fig. 3C) }\end{array}$ & Filtek flow Z-350 & 30 \\
\hline
\end{tabular}

\section{BONDING PROCEDURE}

\section{Conditioning of the Enamel Surface}

The buccal surface of the teeth was polished with pumice slurry using rubber cup mounted on low speed hand piece. After polishing, the teeth were washed with distilled water and dried using oil free air from a 3-way syringe. A total of $37 \%$ orthophosphoric acid was applied to the labial surface and left for a period of 15 seconds. The acid was then washed away with a spray of water for 10 seconds. The tooth surface was then air-dried using oil and moisture free three 3-way syringe until a dull frosty appearance was seen on the surface.

The above procedure was done for all the test specimens, to be bonded with three adhesives to be evaluated. 


\section{Bonding Procedure using Transbond XT: Group 1}

The primer was applied to the etched surface with the help of an applicator brush and cured with light emitting diode (LED) for 10 seconds. The adhesive was then applied to the base of the metal bracket directly from the syringe. The bracket was held and carried to the tooth surface by a bracket holder. The bracket was then positioned on the tooth surface along the midline at a distance of $4 \mathrm{~mm}$ from the occlusal surface. The positioning was achieved with the help of a bracket positioner.

The bracket was pressed on the tooth surface using the reverse end of the bracket holder using uniform finger pressure. The flash around the bracket was then removed with an explorer.

The adhesive was cured using a LED curing unit. A radiometer was used to determine the intensity of the LED. The adhesive was cured from the occlusal, gingival, mesial and distal aspects for 10 seconds each.

The bonded teeth were then kept in distilled water at room temperature for 24 hours before debonding.

\section{Bonding using Esthet-X Flow and Filtek Z-350: Groups II and III}

Bonding procedure for these adhesives was the same as that for group I, but after enamel etching primer step was eliminated.

\section{EVALUATION OF BOND STRENGTH}

SBS was tested with a Universal Testing Machine JJ LLOYD and was evaluated according to the following procedure for all the three samples. The machine has two vertically placed jaws.

- The aluminum block with the tooth embedded was placed in the lower jaw with custom-made jig (fixed head)

- A custom-made chisel was fitted to the upper jaw of the machine (movable head).

At the start of the testing, the jaws are positioned such that the sharp edge of the chisel from the upper block fits under the occlusal wings of the bracket bonded to the tooth. An occlusogingival force was applied to each bracket producing a shear force at the bracket-tooth interface at a crosshead speed of $1 \mathrm{~mm} / \mathrm{min}$.

The JJ LLOYD unit was attached to an electronic console that displayed the debonding forces acting on the bracket tooth interface. Thus, the exact force at which the bracket debonded was noted from the console. This force was expressed in Newtons.
To evaluate the SBS in MPa, from the force value, the following formula was used:

$\begin{aligned} & \text { Shear Bond Strength } \\ & \text { (MPa) }\end{aligned}=\frac{\text { Force in Newtons }}{\text { Area of the bracket base (sq mm) }}$

The area of the bracket base as measured by using digital Vernier calipers $\left(8.68 \mathrm{~mm}^{2}\right)$ (Fig. 4C).

\section{EVALUATION OF THE DEBONDING CHARACTERISTICS}

The debonded tooth surface was examined under an optical microscope at $\times 16$ magnification to determine the bond failure interface (Figs 5A to G).

The adhesive remaining on the bracket base after debonding was scored according to the modified adhesive remnant index (ARI) based on the following guidelines:

- Score $0=$ no adhesive left on bracket

- Score $1=$ less than $25 \%$ of adhesive left on bracket

- Score $2=25 \%$ of adhesive left on bracket

- Score $3=50 \%$ of adhesive left on bracket

- Score $4=75 \%$ of adhesive left on bracket

- Score $5=100 \%$ of adhesive left on bracket.

The values obtained from the SBS testing and the modified ARI scores of the three adhesives were tabulated and the subjected to statistical analysis.

\section{RESULTS}

The results of the SBS and modified ARI scores of the three adhesives: Group I, Transbond XT; group II, Esthet-X flow; group III, Filtek Z-350 flow with their interpretation are presented in Tables 1 and 2 graphically and diagrammatically represented in Graphs 1 and 2A to C.

\section{SHEAR BOND STRENGTH}

- It is evident from the above table that group I has the highest mean SBS $(11.58 \pm 1.3 \mathrm{MPa})$ followed by group III $(11.07 \pm 1.03 \mathrm{MPa})$ and the lowest in group II $(10.70 \pm 2.0 \mathrm{MPa})$.

- One-way ANOVA was used to calculate the p-value ( $<0.05$ was considered significant). One-way ANOVA showed no significant difference in mean SBS among three groups.

- From the above table it was observed that 60 to $70 \%$ of samples in all the three study groups showed score 3.

- Groups II and III showed increased frequency of score 2, 4 and 5 and lower in group I but it was vice versa in score 0 and 1 . 
Comparative Evaluation of the Shear Bond Strength and Debonding Properties of a Conventional Composite

Table 1: Mean and standard deviation and test of significance of shear bond strength (SBS) of the three adhesive groups

\begin{tabular}{ccccccc}
\hline Groups & Samples $(N)$ & Mean SBS $(\mathrm{MPa})$ & Std. dev & Range $(\mathrm{MPa})$ & p-value & F statistic \\
\hline I & 30 & 11.58 & 1.39 & $9.5-14.9$ & & \\
II & 30 & 10.70 & 2.00 & $8.6-19.9$ & 0.0866 & \\
III & 30 & 11.07 & 1.03 & $9.5-12.9$ & & 2.5172 \\
\hline
\end{tabular}

\begin{tabular}{llllllll}
\multicolumn{7}{c}{ Table 2: Frequency distribution of modified ARI of the three adhesive groups } \\
\hline Groups & $\begin{array}{l}\text { Sample } \\
\text { (N) }\end{array}$ & Score 0 & Score 1 & Score 2 & Score 3 & Score 4 & Score 5 \\
\cline { 2 - 7 } & & No.(\%) & No.(\%) & No.(\%) & No.(\%) & No.(\%) & No.(\%) \\
\hline I & 30 & $7(23 \%)$ & $6(20 \%)$ & $4(13 \%)$ & $8(27 \%)$ & $3(10 \%)$ & $2(7 \%)$ \\
II & 30 & $3(10 \%)$ & $4(13 \%)$ & $6(20 \%)$ & $10(34 \%)$ & $4(13 \%)$ & $3(10 \%)$ \\
III & 30 & $3(10 \%)$ & $3(10 \%)$ & $5(17 \%)$ & $10(33 \%)$ & $6(20 \%)$ & $3(10 \%)$ \\
\hline
\end{tabular}

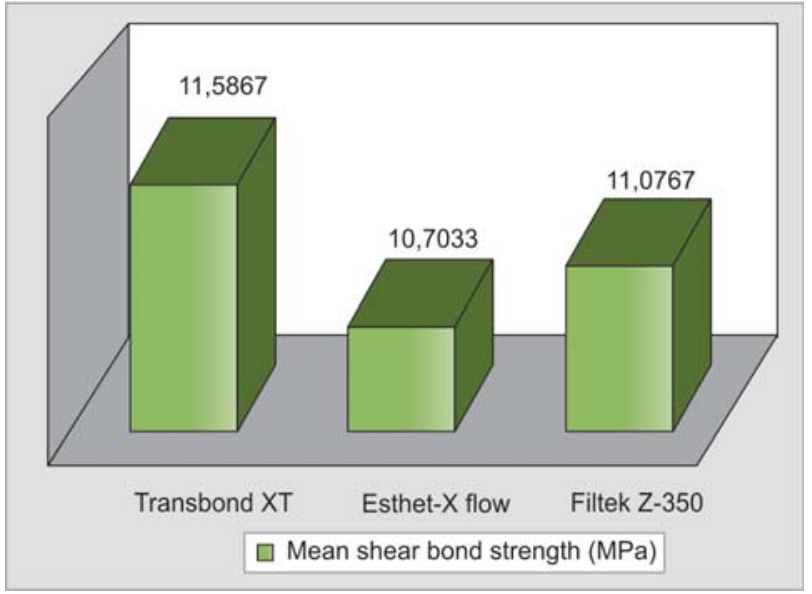

Graph 1: Adhesive remnant index

\section{SUMMARY OF RESULTS}

Under the test conditions, Transbond XT showed highest SBS followed by Filtek Z-350 and the least by Esthet-X flow. However, the difference was not statistically significant when the means of all three study groups were compared and even one-way ANOVA showed no significant difference in mean SBS among three groups.

Frequency distribution of modified ARI for the three adhesive groups showed. Groups II and III showed increased frequency of score 2, 4 and 5 and lower in group I but it was vice versa in score 0 and 1 . All the three groups had ARI score of 3 (60-70\%), suggestive of cohesive type of failure.

\section{DISCUSSION}

The direct bonding of orthodontic brackets has revolutionized and advanced the clinical practice of orthodontics. Present day bonding makes use of acid etchants followed by primer materials as an essential part of the bonding procedure in order to allow good wetting and penetration of the sealant that bond the bracket to the enamel surface. ${ }^{14,15}$

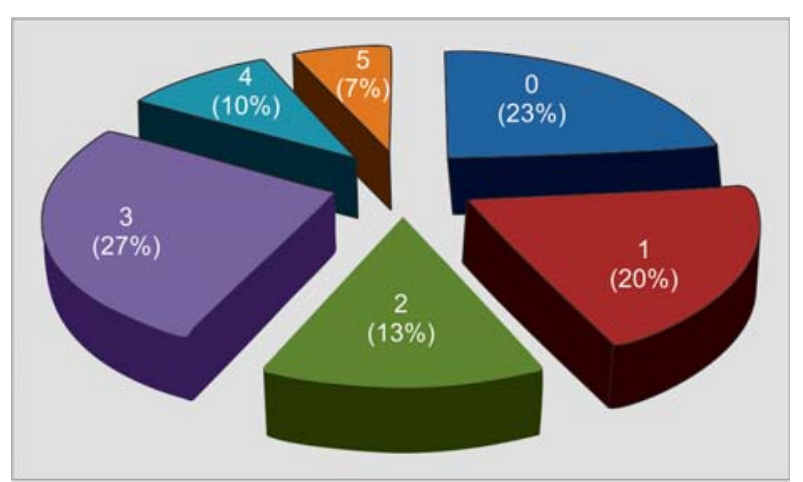

Graph 2A: ARI frequency distribution Transbond XT

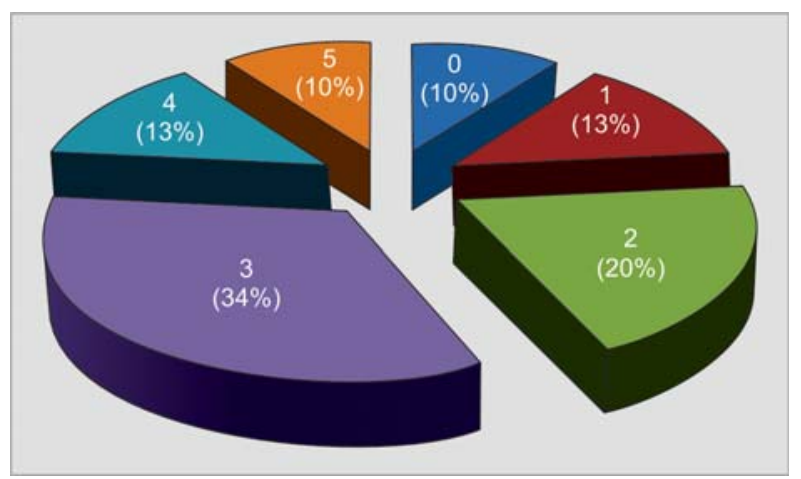

Graph 2B: ARI frequency distribution Esthet-X flow

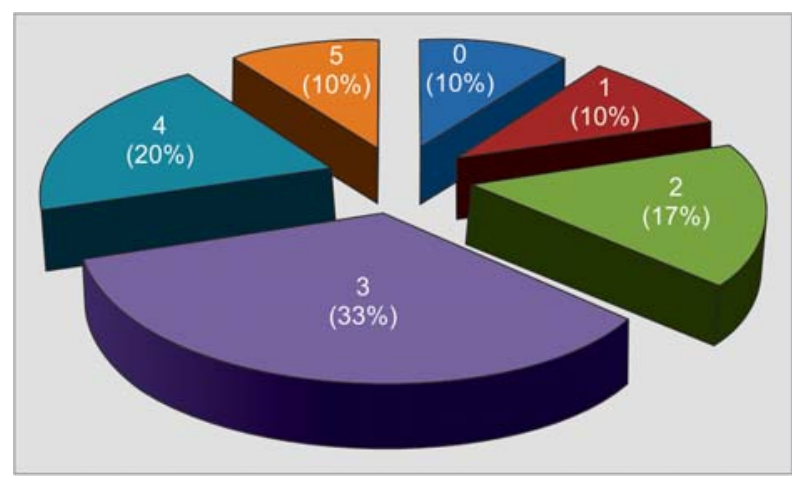

Graph 2C: ARI frequency distribution Filtek Z-350 
The quality of orthodontic treatment is being constantly improved with increasing sophistication of techniques and orthodontic bonding materials that benefits both the patients and the clinician. Advances in material sciences have tried to improve the quality of bonding by refining the composition of bonding materials, dispensing systems and modes of curing, enhancing bond strength, handling characteristics and simplifying the procedure. The main requisite of an orthodontic adhesive is to produce a strong and durable bond to withstand both occlusal and orthodontic force during the course of treatment but at the same time permit bracket removal at the end of treatment without enamel damage and ease of cleaning.

Flowable composites are low viscosity composite resins, created by retaining the same particle size of traditional hybrid composites, but reducing filler content and increasing resin content to reduce viscosity of resin. Flowable composites were marketed for bonding of brackets during early 21st century. Flowable composite merits special attention because of their clinical handling characters of nonstickiness, fluid injectability and SBS comparable to that of traditional composite adhesives.

This study, we therefore undertook which, compare and evaluate effectiveness of Esthet-X flow, and Filtek Z-350 flowable composites will enable the clinician to consider as an alternative orthodontic bonding agent for orthodontics bracket bonding. ${ }^{20}$

The values of the SBS and modified ARI scores obtained for Transbond XT, Esthet-X flow and Filtek Z-350 in the present study were statistically analyzed.

\section{Comparison of SBS of the Three Adhesive Groups}

Transbond XT has a slightly higher mean strength value of $11.58 \pm 1.3 \mathrm{MPa}$ compared to Filtek Z-350 with a mean SBS of $11.07 \pm 1.03 \mathrm{MPa}$ and Esthet-X flow had the lowest SBS value $10.70 \pm 2.0 \mathrm{MPa}$. The differences noted in the mean SBS values of the three adhesives are not statistically significant $(\mathrm{p}=0.086)$. One-way ANOVA was used to calculate the p-value ( $<0.05$ was considered significant). One-way ANOVA showed no significant difference in mean SBS among three groups (Table 1, Graph 1).

Thus, the present study indicated that Transbond XT and Filtek Z-350 have comparable bond strength followed by slightly lower bond strength for Esthet-X flow.

At this juncture it is worthwhile to note that the bond strength of all the three adhesives is quite above the clinically acceptable level of 5.9 to $7.8 \mathrm{MPa}$ as suggested by Reynolds. ${ }^{19}$ Lopez $^{21}$ recommended a value of $7 \mathrm{MPa}$ as minimum bond strength for successful clinical bonding.
According to Profit, ${ }^{22}$ forces of mastication are highly variable which ranges upto $50 \mathrm{~kg}$ and orthodontic forces in the range of 50 to $150 \mathrm{gm}$.

Among the various commercially available BisGMAbased resins Transbond XT has been extensively evaluated for its own bond strength as well as a adhesive to evaluate other factors influencing bond strength namely enamel conditioning method, methods of polymerization, bracket types (metal or ceramic) and different debonding methods.

Olsen, Owens Jr, Bishara, Oesterle and Tecco et al to name a few have reported higher SBS values for Transbond $\mathrm{XT}$ when evaluated under different testing conditions as observed in the present study. ${ }^{23}$

Simona $^{24}$ and Attilio ${ }^{19}$ have reported very high values of 23.23 and 23.47 MPa for Transbond XT and 34.80 and 24.98 MPa for flowable composites compared to 11.64 and 11.0 MPa expressed in the present study. They suggested that flowable composites can be used for orthodontic bonding.

Dong-Bum-Ryou ${ }^{25}$ reported a lower SBS value for flowable composites compared to that of Admira Flow in the present study and he concluded that flowable composites with no intermediate bonding resin could be conveniently applied for orthodontic bracket.

However, Tancan Uysal ${ }^{26}$ reported a very low value for flowable composites ranging from 6 to $8 \mathrm{MPa}$ compared to 17.10 MPa showed for Transbond XT and concluded that flowable composites are not suitable for orthodontic bonding.

The goal of current orthodontic research is to improve the bonding procedure by reducing chairside time during bonding and debonding without jeopardizing the ability to maintain clinically acceptable bonds strength. Clinicians are interested in determining the level at which the bond failure will take place with force application.

According to Fox, ${ }^{27}$ and Eliades ${ }^{28}$ mean bond strength and standard deviation may not be the best indicators for evaluating the bond strength of the bonding materials. When considering the bond failure, weaker values (tail of the distribution) may be more important. Therefore it may be more appropriate to use a function that relates probability of failure to applied force. It is suggested that in vitro and in vivo bond strength testing should include some form of survival analysis. This gives the clinician a better idea of how the material or bracket is likely to perform in the clinical situation. Therefore in addition to ANOVA, which considers only mean and standard deviation, Kaplan-Meier survival analysis was also performed to analyze the data because it considers the entire spread of the data and thus helps to evaluate the clinical performance better. 


\section{Frequency Distribution of modified ARI Scores of} the Three Adhesive Groups

The frequency distribution of the modified ARI scores reveals that 60 to $70 \%$ of the samples in all the three study groups showed score 3 suggestive of a cohesive fracture (Table 2, Graphs 2A to C).

Ideal ARI score is a subject of debate. Proponents of low score of modified ARI (score 1 and 2) claim that the tooth surface requires extensive cleaning of already damaged etched enamel surface. ${ }^{29}$ Group I had more percentage of score 1and 2 compare with that of groups II and III.

Proponents of high score of modified ARI (score 4 and 5) claims that there is no need of extensive cleaning, but there is a possibility of enamel crack or tear since entire adhesive comes out with bracket base. Groups II and III showed increased frequency of score 2, 4 and 5 compare to group I.

It is suggested that bond failure at bracket adhesive interface is more desirable than adhesive enamel interface, because enamel fracture and cracking have been reported, though inherent weakness of the tooth surface can also cause tooth fracture.

The cohesive fracture noted in the present study is favorable and indicates moderate amount of bond strength at clinically acceptable level, and facilitate easy debonding after treatment. Further, it requires minimal clean up and less damage during debonding.

The above discussion on the present study reveals that Filtek Z-350 has comparable bond strength with that of Transbond XT and Esthet-X flow has slightly lower bond strength values. However, the SBS values of all the three adhesives are within the clinically acceptable levels and they exhibit cohesive type of bond failure. Probability of failure rate is almost similar for all the three adhesives, the difference being not statistically significant.

Both flowable composites on the other hand have a decreased filler particle, i.e. it flows while being applied and contoured but remains firm when stationary. However, when this material was used for bonding in the present study drifting of the brackets was noticed due to its increased flowability and required extra care to position the brackets.

It is evident from the present study that flow property and viscosity of adhesive plays an important role. Within certain limits, thinner and flowable adhesive paste will facilitate better penetration of the adhesive into mesh of the bracket base and the microporosities of the etched enamel surface and improve handling properties and bond strength. Therefore, a balance is required between the flow and viscosity to obtain optimal consistency to achieve optimal bond strength and improve handling property.

Though Transbond XT is a clinically efficient material as again confirmed from this study, Esthet- $X$ flow and Filtek Z-350 flow, if their flow and viscosity are balanced to improve handling property can definitely be considered as an alternative bonding system due to its comparable bond strength and debonding characters and other reported properties of biocompatibility. Considering the in vivo nature of the present study the findings should be interpreted with caution while applying it for clinical application. The efficacy of the flowable composites as a bonding agent needs in vivo and clinical assessment through a survival analysis. Preliminary evaluation done in this study however will be a valuable guide for future in clinical use.

\section{CONCLUSION}

The following conclusions are drawn from this comparative in vitro study on the SBS and debonding characters of Transbond XT, Esthet-X flow and Filtek Z-350 using metal brackets.

- Filtek Z-350 has a comparable mean bond strength value $(11.07 \pm 1.03 \mathrm{MPa})$ like that of Transbond XT (11.58 \pm $1.3 \mathrm{MPa})$.

- Esthet-X flow has slightly lower mean bond strength value $(10.70 \pm 2.0 \mathrm{MPa})$ compared Filtek Z-350 and Transbond XT.

- All the three adhesives exhibited clinically acceptable bond strength.

- 60 to $70 \%$ of all the three adhesives exhibited cohesive type of bond failure.

- The efficacy of the flowable composites as a bonding agent needs in vivo and clinical assessment through a survival analysis.

- Filtek Z-350 and Esthet-X flow exhibited problems with handling properties and flowability. Drifting of brackets was noted and pressure was needed for positioning the bracket with Filtek Z-350 and Esthet-X flow.

- The flow property and viscosity should be balanced to obtain optimal consistency to achieve optimal bond strength and improve handling property for Filtek Z-350 and Esthet-X flow.

- Future research need to be focused toward the biocompatibility, rheological property and viscosity for Filtek Z-350 and Esthet-X flow to be used as orthodontic adhesive.

\section{MANUFACTURER NAME}

- 3M UNITEK, Monrovia, California

- Dentsply 
- Gemini, 3M Unitek, Monrovia, California

- Universal testing machine JJ LLOYD $20 \mathrm{KN}$

- Stereomicroscope microscope.

\section{REFERENCES}

1. Buonocore MG. A simple method of increasing the adhesion of acrylic filling materials to enamel surfaces. J Dent Res 1955;34:849-53.

2. Newman GV, Synder WH, Wilson CW. Acrylic adhesives for bonding attachments to tooth surfaces. Angle Orthod 1968;38:12-18.

3. Retief DH, Dreyer CJ, Gavron G. The direct bonding of orthodontic attachment to teeth by means of an epoxy resin adhesive. Am J Orthod 1970;58:21-40.

4. Thanos CE, Munholland T, Caputo AA. Adhesion of mesh base direct bonding brackets. Am J Orthod 1979;75:421-23.

5. Gorelik L. Bonding metal brackets with self polymerizing sealant composite: A 12 month assessment. Am J Orthod 1977;71: 542-53.

6. Kugel G, Ferrari M. The science of bonding from first to sixth generation. J Am Dent Assoc 2000;131:205-45.

7. Brosnihan J, Safranek L. Orthodontic bonding: The next generation. J Clin Orthod 2000;34(10):614-16.

8. Chigira H, Koike T, Hasegawa T, Itoh K, Wakumoto S, Hyakawa T. Effect of the self-etching dentin primers on the bonding efficacy of dentin adhesive. Dent Mater J 1989;8: 86-92.

9. Nishida K. Development of a new bonding system [Abstract]. J Dent Res 1993;72:137.

10. Nakabayashi N. Dentinal bonding mechanisms. Quintessence Int 1991;22:73-74.

11. Eliades T, Eliades G, Brantley WA, Johnston WM. Residual monomer leaching from chemically cured and visible light cured orthodontic adhesives. Am J Orthod Dentofacial Orthop 1995;108(3):316-21.

12. Tell RT, Sydiskis RJ, Isaacs RD, Davidson WM. Cytotoxicity of direct bonding adhesives. Am J Orthod Dentofacial Orthop 1988;93:419-22.

13. Li Y, Noblitt TW, Dunipace AJ, Stookey GK. Evaluation of mutagenicity of restorative dental materials using the Ames Salmonella/ Microsome test. J Dent Res 1990;69(5):1188-92.

14. Fredricks HE. Mutagenic potential of orthodontic bonding materials. Am J Orthod Dentofacial Orthop 1981;80(3):316-24.

15. Tarumi H, Imazato S, Narimatsu M, Matsuo M, Ebisu S. Estrogenicity of fissure sealants and adhesive resins determined by reporter gene assay. J Dent Res 2000;79(11):1838-43.

16. Altuna G, Freeman E. The reaction of skin to primers used in the 'single-step' bonding systems. Am J Orthod Dentofacial Orthop 1987;91:105-10.

17. Terhune WF, Sydiskis RJ, Davidson WM. In vitro cytoxicity of orthodontic bonding materials. Am J Orthod Dentofacial Orthop 1983;83(6):501-06.

18. Elaut J, Asscherickx K, Vande Vannet B, Wehrbein H. Flowable composites for bonding lingual retainers. J Clin Orthod 2002;36:597-98.
19. D’Attilio M, Traini T, Di lorio D, Varvara G, Festa F, Tecco S. Shear bond strength, bond failure, and scanning electron microscopy analysis of a new flowable composite for orthodontic use. Angle Orthod 2003;75(3):410-15.

20. Anusavica KJ, Phillips RW. Phillips science of dental material (11th ed). Saunders, 2003.

21. Lopez JI. Retentive shear strength of various binding attachment base. Am J Orthod Dentofacial Orthop 1980;77:669-78.

22. Proffit. In vivo bonding of glass ionomer cements. J Dent Res 1986; vol 62:571-74.

23. Tecco S, Traini T, Caputi S, Festa F, Luca V, D’Attilio M. A new one-step dental flowable composite for orthodontic use: An in vitro bond strength study. Angle Orthod 2005;75:672-77.

24. Wataha JC, Lockwood PE, Bouillaguet S, Noda M. In vitro biological response to core and flowable dental restorative materials. Dent Mater 2003;19(1):25-31.

25. Ryou DB, Park HS, Kim KH, Kwon TY. Use of flowable composite for orthodontic bracket bonding. Angle Orthod 2008;78(6):1105-09.

26. Uysal T, Sari Z, Demir A. Are the flowable composites suitable for orthodontic bracket bonding? Angle Orthod 2004;74:5; 697-702.

27. Fox NA, McCabe FJ, Buckley JG. A critique of Bond strength testing in Orthodontics. Br J Orthod 1994;21:33-43.

28. Eliades T, Brantley WA. The inappropriateness of conventional orthodontic bond strength assessment protocols. Eur J Orthod 2000;22:13-23.

29. Byrant S, Retief DA, Russel CM. Tensile bond strength of orthodontic bonding resin and attachments to etched enamel surface. Am J Orthod Dentofacial Orthop 1987;92:255-31.

\section{ABOUT THE AUTHORS}

\section{S Pradeep (Corresponding Author)}

Senior Lecturer, Department of Orthodontics and Dentofacial Orthopedics, JSS Dental College and Hospital, Mysore, Karnataka India, Phone: +91 09886785810, e-mail: pradeeps1983@gmail.com

\section{Ravi Shanthraj}

Professor, Department of Orthodontics and Dentofacial Orthopedics JSS Dental College and Hospital, Mysore, Karnataka, India

\section{H Jyothi Kiran}

Professor, Department of Orthodontics and Dentofacial Orthopedics JSS Dental College and Hospital, Mysore, Karnataka, India

\section{BM Shivalinga}

Professor and In-Charge of PG Studies, Department of Orthodontics and Dentofacial Orthopedics, JSS Dental College and Hospital Mysore, Karnataka, India 